This is an author produced version of a paper published in Journal of Chemical Ecology. This paper has been peer-reviewed and is proofcorrected, but does not include the journal pagination

Citation for the published paper:

Becher, P.G., Bengtsson, M., Hansson, B.S., Witzgall, P. (2010) Flying the fly: Long-range flight behavior of drosophila melanogaster to attractive odors. Journal of Chemical Ecology. Volume: 36 Number: 6, pp 599-607. http://dx.doi.org/10.1007/s10886-010-9794-2

The original publication is available at www.springerlink.com. Access to the published version may require journal subscription.

Published with permission from: Springer-Verlag.

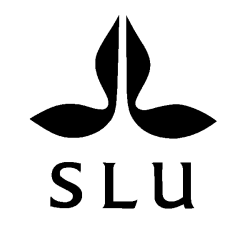

Epsilon Open Archive http://epsilon.slu.se 


\section{Flying the Fly: Long-range Flight Behavior of Drosophila melanogaster to Attractive Odors}

Paul G. Becher • Marie Bengtsson • Bill S. Hansson • Peter Witzgall

P. G. Becher $(\square) \bullet$ M. Bengtsson • P. Witzgall

Chemical Ecology Group, SLU, 23053 Alnarp, Sweden

e-mail: paul.becher@ltj.slu.se

B. S. Hansson

Department of Evolutionary Neuroethology, Max Planck Institute for Chemical Ecology, Jena, Germany 


\begin{abstract}
The fruit fly, Drosophila melanogaster Meigen (Diptera: Drosophilidae), is a model for how animals sense, discriminate, and respond to chemical signals. However, with $D$. melanogaster information on the behavioral activity of olfactory receptor ligands relies largely on close-range attraction, rather than on long-range orientation behavior. We developed a flight assay to relate chemosensory perception to behavior. Headspace volatiles from vinegar attracted $62 \%$ flies during a $15-\mathrm{min}$ experimental period. Flies responded irrespective of age, sex, and mating state, provided they had been starved. To identify behaviorally relevant chemicals, we compared attraction to vinegar with synthetic chemicals. Stimuli were applied by a piezoelectric sprayer at known and constant release rates. Re-vaporized methanol extracts of Super Q trapped vinegar volatiles attracted as many flies as vinegar. The main compound acetic acid elicited significant attraction as a single compound. Two other vinegar volatiles, 2-phenyl ethanol and acetoin, produced a strong synergistic effect when added to acetic acid. Geosmin, a microbiological off-flavor, diminished attraction to vinegar. This wind tunnel assay based on a conspicuous and unambiguous behavioral response provides the necessary resolution for the investigation of physiologically and ecologically relevant odors and will become an essential tool for the functional analysis of the D. melanogaster olfactory system.
\end{abstract}

Keywords Acetic acid - Drosophila melanogaster - Flight behavior • Fruit fly • Geosmin $\cdot$ Long-range attraction $\cdot$ Olfactory system $\cdot$ Vinegar fly $\cdot$ Wind tunnel $•$ 


\section{Introduction}

What triggers take-off and upwind flight behavior in an insect? Two types of flight initiation are distinguished in the fruit fly, Drosophila melanogaster Meigen (Diptera: Drosophilidae) - a fast escape in response to threatening visual stimuli and a slow voluntary take-off based on the assessment of a stimulus, in reflection of the internal physiological state of the animal (Trimarchi and Schneiderman 1995; Allen et al. 2006; Card and Dickinson 2008). Olfactory cues elicit premeditated flights. Following take-off, the odor signal mediates upwind oriented flight and guides the insect towards the source (Budick and Dickinson 2006; Chow and Frye 2008).

Molecular, genetic, and neurosensory knowledge in D. melanogaster frames the most complete picture of an olfactory system that is available, from odorant receptor genes to neuroanatomy and central processing of the olfactory input (Couto et al. 2005; Benton et al. 2007; Jefferis et al. 2007; Dickson 2008). A current challenge is to further expand our view to also include odor-mediated behavior that reflects the entire integration pathway from ligand-binding to motor output. A distinct behavior such as the voluntary initiation of odor-mediated flight is an opportunity to investigate how the fly processes and evaluates chemical signals that evoke the decision to fly into the scented wind.

Towards this goal, we need to improve our knowledge of the behavioral effect of olfactory receptor ligands in D. melanogaster. This relies largely on screening of compounds by extracellular recordings and imaging of the input of olfactory receptor neurons into the antennal lobe. The response of $D$. melanogaster to odors is usually studied in trapping or oviposition assays, using small containers, in spite of common long-range orientation to odor sources in nature. Clearly, small dimensions, the lack of an air-flow and long test duration lead to an elevated response to suboptimal stimuli and limit the discriminative power and sensitivity of such experiments (Vosshall and Stocker 2007). In addition, the chemicals used as olfactory stimuli in current molecular and neurophysiological studies of the D. melanogaster olfactory system do not necessarily match the stimuli used by flies for location of food sources and oviposition sites. 
Wind tunnels have been employed to investigate odor-mediated upwind flight behavior in D. melanogaster (Budick and Dickinson 2006), but have, with the exception of the pheromone compound cis-vaccenyl acetate (Bartelt et al. 1985), not been used to identify behavior-modifying chemicals. We have set up a wind tunnel to study long-range attraction in relation to odor quality and the internal physiological state of the flies. After measuring fly attraction to vinegar and some of its key chemical components, we investigated the effect of fly age, sex, mating state, and starvation. The results demonstrate that a flight tunnel is a sensitive instrument for the analysis of odor-mediated behavior in D. melanogaster.

\section{Methods and Materials}

Insects An Oregon $\mathrm{R}$ strain of the fruit fly, D. melanogaster, was reared on a standard sugar-yeast-cornmeal diet at room temperature $\left(19-22^{\circ} \mathrm{C}\right)$ and under a 8:16 h L:D photoperiod. Newly eclosed flies were removed from the diet daily. Adult flies were either kept in 30-ml plexiglass vials on fresh diet, or were starved up to $3 \mathrm{~d}$ on a humidified piece of cotton wool. For tests with mated males or females, newly emerged test flies were kept together with an excess ratio (ca. 1.5:1) of older virgin flies (ca. 1 wk old) of the opposite sex. Flies were anesthetized with $\mathrm{CO}_{2}$ and sexed $24 \mathrm{hr}$ before the experiment. For tests with unmated insects, newly emerged flies were sexed up to $3 \mathrm{hr}$ after eclosion. Exposure to $\mathrm{CO}_{2}$ did not exceed $5 \mathrm{~min}$ and preparatory control experiments (data not shown) as well as our subsequent flight assays (e.g., Fig. 2b) did not indicate an adverse effect of anesthetization on fly behavior. The flies were tested during the last $3 \mathrm{hr}$ of the photophase.

Headspace Collection, Chemical Analysis, and Chemicals Volatiles were collected from ca. $70 \mathrm{ml}$ balsamic vinegar (Aceto balsamico di Modena, Urtekram, Denmark, aged at least three years) by blowing charcoal-filtered air $(0.91 / \mathrm{min})$ with an aquarium pump through the vinegar in a 1-1 gas wash bottle, exiting through a Super Q trap at the gas outlet. The volatile trap was made of a $4 \times 40-\mathrm{mm}$ glass tube containing 35 mg Super Q (80/100 mesh; Alltech, Deerfield, IL, USA) held between glass wool plugs (Tasin et al. 2006). Before use, the trap was rinsed with $3 \mathrm{ml}$ of 
methanol (redistilled $>99.9 \%$ purity, Merck, Darmstadt, Germany) and $n$-hexane (redistilled $>99.9 \%$ purity; Labscan, Malmö, Sweden). After 30 min of collecting vinegar odors, compounds were eluted with $0.3 \mathrm{ml}$ of redistilled methanol. The samples were analyzed on a gas chromatograph coupled to a mass spectrometer (GCMS; 6890 GC and 5975 MS, Agilent Technologies Inc., Santa Clara, CA). The gas chromatograph was equipped with a DB-Wax or a HP-5MS fused silica capillary column $(30 \mathrm{~m} \times 0.25 \mathrm{~mm} \times 0.25 \mu \mathrm{m}$ film thickness; J\&W Scientific, Folsom, California, Agilent). The amounts of acetic acid, 2-phenyl ethanol, acetoin, and ethyl acetate in the samples were quantified in comparison with synthetic standards (Sigma-Aldrich, Stockholm, Sweden). Ethyl acetate and ethanol were quantified in a headspace collection eluted with pentane. The methanol filter eluates were revaporized in the wind tunnel by the piezoelectric sprayer (see below).

Wind Tunnel Most of our flight assays were conducted in a wind tunnel, which was built of glass and had a flight section of $30 \mathrm{~cm} \times 30 \mathrm{~cm} \times 100 \mathrm{~cm}$. The airstream in the tunnel $(0.25 \mathrm{~m} / \mathrm{s})$ was produced by a fan (Fischbach $\mathrm{GmbH}$, Neunkirchen, Germany), which blew air into the tunnel through an array of four activated charcoal cylinders (14.5 cm diam. x $32.5 \mathrm{~cm}$ long; Camfil, Trosa, Sweden). A $30 \mathrm{~cm}$ x $30 \mathrm{~cm}$ x $30 \mathrm{~cm}$ compartment at the upwind end of the tunnel held the piezoelectric sprayer (see below) and was separated from the flight section by a polyamide mesh (pore size $0.5 \mathrm{~mm} \times 0.5 \mathrm{~mm}$; Sintab, Oxie, Sweden). The downwind end of the tunnel was closed by the same mesh. The tunnel was lit diffusely from above and light intensity inside the wind tunnel was 13 lux. Temperature ranged from 19 to $22^{\circ} \mathrm{C}$; relative humidity from 35 to $50 \%$.

The speed of the airstream was selected based on preliminary tests of the response of the flies. Significantly more flies (2-d-old, starved for $2 \mathrm{~d}$ ) flew to the standard stimulus vinegar at $0.25 \mathrm{~m} / \mathrm{s}(62 \pm 10 \%$ attraction $)$ and $0.15 \mathrm{~m} / \mathrm{s}(54 \pm 9 \%$ attraction) than at a lower speed of $0.05 \mathrm{~m} / \mathrm{s}(40 \pm 11 \%$; ANOVA, $F=6.20, \mathrm{df}=14$, $P<0.05)$.

Odor Delivery Charcoal-filtered air was blown through the wash bottle with balsamic vinegar (similar as described above but without air filter) or distilled water 
(MilliQ, Millipore, Bedford, MA, USA), into a teflon tube $(0.5 \mathrm{~cm}$ diam.), leading to an attached Pasteur pipette. The pipette was facing downward into a wide-mouth 225-ml glass jar (38 mm diam.). The air stream containing the stimulus (e.g., volatiles of authentic vinegar or distilled water) emanated as a wide plume from the opening of the jar, in the center of the upwind end of the tunnel. The shape of the odor plume was verified with titanium oxide. Flies were scored when landing at the tip of the pipette, at the top edge of the jar, or inside the jar. All glassware was heated to $375^{\circ} \mathrm{C}$ during $8 \mathrm{hr}$ before use.

Headspace samples and solutions of synthetic compounds were released from a piezoelectric sprayer. A microinjection pump (CMA Microdialysis AB, Solna, Sweden) delivered the solutions at a rate of $10 \mu \mathrm{l} / \mathrm{min}$ from a $1-\mathrm{ml}$ syringe, through teflon tubing, to a $25-\mu 1$ glass capillary tube with an elongated tip. A piezo-ceramic disc (Valvo, Hamburg, Germany) vibrated the glass capillary at ca. $200 \mathrm{kHz}$ and thus produced an aerosol that evaporated from the tip of the capillary (El-Sayed et al. 1999). The capillary was placed at the vertical midline, $20 \mathrm{~cm}$ from the floor, at the upwind end of the tunnel. The dimension of a hexane plume was measured at the downwind end of the tunnel with a photoionization detector (ppbRAE Plus, Scantec, Sävedalen, Sweden). The plume was approximately in the center of the tunnel, and its cross-section was oval, with a vertical axis of ca. $12 \mathrm{~cm}$ and a horizontal axis of ca. $7 \mathrm{~cm}$. The piezoelectric sprayer ensured application of odor solutions at a constant rate and known chemical purity. A glass cylinder (60 x $95 \mathrm{~mm}$ diam.) covered with a metal mesh (pore size $2 \mathrm{~mm}$ ) shielded the vibrating capillary from flies and mechanical damage. When the sprayer was used to deliver the stimuli for the flight assay, flies were scored when landing at the metal mesh.

Initially, four solvents (Table 1) were assayed in the wind tunnel to help to choose an appropriate solvent that would be efficient to elute and dissolve volatile organic compounds without attracting fruit flies by itself. Distilled water (MilliQ) and the redistilled organic solvents methanol, hexane, and heptane (redistilled $>99.9 \%$ purity, Labscan) were delivered at $10 \mu 1 / \mathrm{min}$ (Table 1). Flies were not attracted to the non-polar solvents hexane and heptane, but these were not suitable for dissolving acetic acid, which is the major headspace component of vinegar. 
Methanol was a suitable solvent for acetic acid and other vinegar compounds and was used in subsequent tests.

Table 1 Upwind flight and landing of Drosophila melanogaster in a wind tunnel in response to solvents delivered from a piezoelectric sprayer

\begin{tabular}{lc}
$\begin{array}{l}\text { Solvent } \\
(10 \mu 1 / \mathrm{min})\end{array}$ & $\begin{array}{c}\text { Flies landing at source } \\
(\% \pm \mathrm{SD})^{\mathrm{c}}\end{array}$ \\
\hline Water & $6 \pm 9 \mathrm{a}$ \\
Methanol & $4 \pm 2 \mathrm{a}$ \\
Hexane & $0 \mathrm{a}$ \\
Heptane & $0 \mathrm{a}$ \\
\hline${ }^{\mathrm{a}}$ Flies were 2-d-old and starved. \\
${ }^{\mathrm{b}}$ Percentages $(N=5$ batches of 20 flies) followed by \\
different letters indicate significant differences \\
$\begin{array}{l}\text { (Tukey's test following ANOVA; } F=2.12, \text { df }=19, \\
P=0.138) .\end{array}$
\end{tabular}

Trapping Study of Diel Flight Period in the Laboratory A trap was made of a 225$\mathrm{ml}$ capped glass jar filled with $25 \mathrm{ml}$ of a $0.1-\%$ Triton X-100 (Tamro Medlab AB, Mölndal, Sweden) solution in distilled water. It contained a $30-\mathrm{ml}$ plastic vial that was filled with the bait, balsamic vinegar (the same as used in the wind tunnel assay and chemical analyses) or a macerated banana-water mixture. Distilled water was the control. A plastic lid holding a cut pipette tip allowed the odor to emanate and provided a trap entry for the flies. Traps were placed in plastic trays filled with water. Five replicates of three different trap lures were randomly distributed in the laboratory. Seventy 1- to 3-d-old flies were released into the wind tunnel room $(N=3), 4 \mathrm{hr}$ after onset of the photophase, under a 8:16 L:D photoperiod. The traps were checked every $2 \mathrm{hr}$ during the photoperiod.

Test Protocol and Statistical Analyses For wind tunnel assays, batches of 18 to 24 flies were allowed to walk or fly into a $225-\mathrm{ml}$ glass jar (12 cm high) that was then closed with a screw cap and covered with tin foil. The jar was exposed to the odor plume at the downwind end of the tunnel. A plug closing a $10-\mathrm{mm}$ hole in the middle of the screw cap was removed to allow the flies to exit. The test period was $15 \mathrm{~min}$. Variances of the mean attraction $(N=5)$ were checked for homogeneity ( $F$ test or 
Bartlett's test) and analyzed statistically by a $t$-test or Tukey's test following an analysis of variance (ANOVA). For the trapping study in the laboratory differences between the two baits and differences between sexes were analyzed by a t-test.

\section{Results}

Attraction to Vinegar Headspace Volatiles and Synthetic Vinegar Compounds in the Wind Tunnel Starved, 2-d-old flies were strongly attracted to authentic vinegar headspace emanating from the wash bottle $(50 \%$ of the test flies reached the source after $9.0 \pm 2.8 \mathrm{~min}$ and overall attraction was $62 \pm 10 \%$ within the $15-\mathrm{min}$ experimental period, Fig. 1). The quantitative assessment of vinegar headspace volatiles revealed that acetic acid was the most abundant compound (Fig. 1a) It was released from the wash bottle at $17.4 \mu \mathrm{g} / \mathrm{min}$ (GC-MS). Re-vaporized (sprayed) filter eluate of vinegar headspace collections, releasing acetic acid at the same rate, attracted $74 \pm 14 \%$ flies (Fig. 1c).

Three known ligands of $D$. melanogaster olfactory receptor neurons, acetoin, ethyl acetate, and 2-phenyl ethanol (Stensmyr et al. 2003; Hallem and Carlson 2006; De Bruyne and Baker 2008), were also found in vinegar headspace (Table 2; Cocchi et al. 2008; Guerrero et al. 2007). They were tested as single compounds and in blends, at the release rate found in vinegar headspace. Although not tested individually it is noteworthy that ethanol was present among the headspace volatiles, but only at $1.5 \%$ of the amount of acetic acid (Table 2). Acetic acid, which was attractive alone, was also required in the blends to elicit attraction, whereas the response to the 3-component blend of 2-phenyl ethanol, acetoin, and ethyl acetate did not cause significan attraction (Table 2). Even at a release rate of $17.4 \mu \mathrm{g} / \mathrm{min}$ (i.e., the same as for acetic acid) the single compounds were not significantly attractive by themselves; acetoin attracted $1 \pm 2 \%$, ethyl acetate $3 \pm 4 \%$, and 2-phenyl ethanol $6 \pm 5$ $\%$ flies $(N=5)$. A synergistic effect on fly attraction was produced by adding 2 phenyl ethanol to acetic acid, and by adding acetoin to these two compounds. Admixture of ethyl acetate to the 3-component blend further enhanced the fly response, but the difference between the 3- and 4-component blend was not significant (Table 2). 

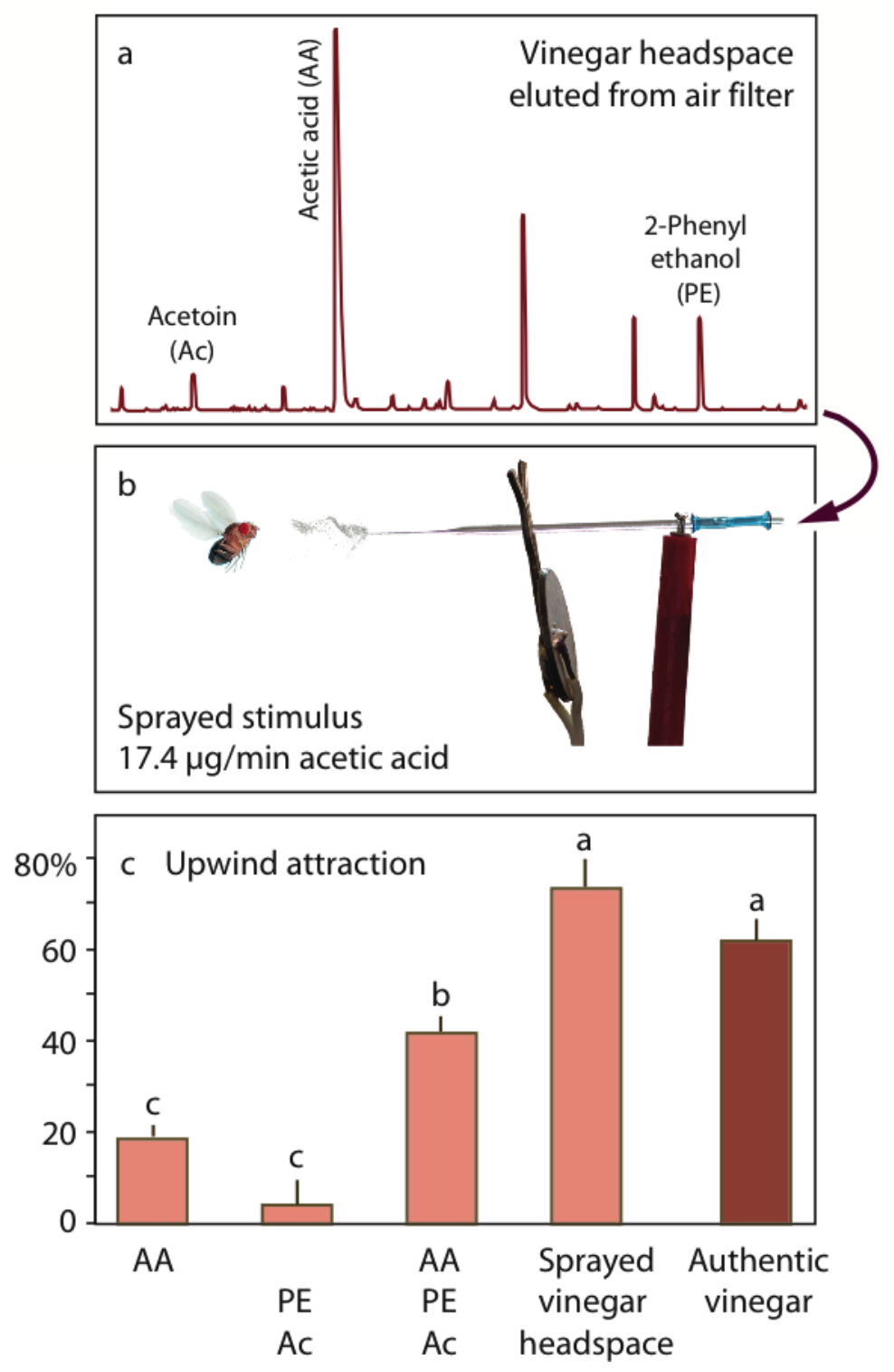

Fig. 1 Attraction of 2-d-old starved Drosophila melanogaster adults in a wind tunnel to GC-MS analyzed (a) and defined odor samples that were released from a glass capillary attached to a piezo-ceramic disk (piezoelectric sprayer) (b). The release rates of acetic acid (AA), the main vinegar compound $(17.4 \mu \mathrm{g} / \mathrm{min})$, ethyl acetate $(0.103 \mu \mathrm{g} / \mathrm{min}$ ), acetoin (Ac; $0.58 \mu \mathrm{g} / \mathrm{min})$ and 2-phenyl ethanol (PE; $1.22 \mu \mathrm{g} / \mathrm{min})$ in sprayed headspace and in synthetic blends mimic vinegar headspace. Flies were attracted to sprayed synthetic vinegar compounds, sprayed vinegar headspace extracted from air filters and to authentic vinegar headspace from a wash bottle (c). Different lowercase letters in (c) indicate significant differences according to ANOVA followed by Tukey's test $(N=5$ batches of 20 flies; $F=50.61 ; \mathrm{df}=24$, $P<0.001$; error bars show standard deviation of the mean). 
Table 2 Attraction of Drosophila melanogaster to synthetic vinegar compounds and to vinegar headspace volatiles delivered by a piezoelectric sprayer

\begin{tabular}{|c|c|c|c|c|c|c|c|c|c|c|c|c|c|c|c|c|c|}
\hline \multicolumn{18}{|c|}{ Compound $(\mu \mathrm{g} / \mathrm{min})^{\mathrm{a}}$} \\
\hline Acetic acid & 17.4 & - & & & & • & - & • & & & & - & - & - & & • & • \\
\hline 2-Phenyl ethanol & 1.22 & & - & & & - & & & - & • & & - & - & & - & - & • \\
\hline Acetoin & 0.58 & & & • & & & • & & • & & • & • & & • & • & • & • \\
\hline Ethyl acetate & 1.03 & & & & • & & & • & & • & - & & • & - & - & - & • \\
\hline Other compounds ${ }^{\mathrm{b}}$ & 6.80 & & & & & & & & & & & & & & & & • \\
\hline Attraction $(\%)^{\mathrm{c}}$ & & $19 \mathrm{de}$ & 8 ef & $2 \mathrm{f}$ & $0 \mathrm{f}$ & $31 \mathrm{~cd}$ & $10 \mathrm{ef}$ & $2 \mathrm{f}$ & $4 \mathrm{f}$ & $0 \mathrm{f}$ & $3 \mathrm{f}$ & $42 \mathrm{bc}$ & $17 \mathrm{e}$ & $16 \mathrm{e}$ & $9 \mathrm{ef}$ & $52 \mathrm{~b}$ & $74 \mathrm{a}$ \\
\hline
\end{tabular}

${ }^{\mathrm{a}}$ Release rates mimic a headspace collection of vinegar (last column).

${ }^{\mathrm{b}}$ Other compounds ( $>1 \%$ relative to acetic acid) found in vinegar headspace, according to GC-MS analysis and in agreement with literature (Zeppa et al. 2002; Guerrero et al. 2007; Cocchi et al. 2008) included: ethanol (1.5\%), 3-methyl-1-butanol (2.1\%), 2,3butanediol diacetate $(1.7 \%)$, acetyl furan $(1.0 \%)$, 2-methyl-propanoic acid $(1.0 \%), 5$ methyl-2-furancarboxyaldehyde (2.6\%), isovaleric acid (17.9\%), 2-phenylethyl acetate $(6.8 \%)$, hexanoic acid $(2.3 \%)$, and octanoic acid (2.1\%)

${ }^{c}$ Percentages ( $N=5$ batches of 20 flies) followed by different letters are significantly different (ANOVA followed by Tukey's test, $F=57.920 ; \mathrm{df}=15,64 ; \mathrm{P}<0.001$ ).

Threshold Concentration of Orientation to Vinegar in the Wind Tunnel A doseresponse test showed that re-vaporized vinegar headspace samples elicited significant attraction at release rates ranging over three magnitudes (Table 3). The release rate of acetic acid from pure vinegar was $17.4 \mu \mathrm{g} / \mathrm{min}$ as measured with air filters, but flies were still attracted to a 1000-fold diluted headspace collection, releasing as little as $17.4 \mathrm{ng} / \mathrm{min}$ acetic acid. This agrees with the significant attraction to a 1000 -fold dilution of vinegar in water. In contrast, the response to a 1:10 dilution of acetic acid alone was not different from blank, corroborating the synergistic effect of the other vinegar compounds (Tables 2 and 3 ). 
Table 3 Attraction of Drosophila melanogaster to different release rates of acetic acid and vinegar

\begin{tabular}{cccc}
\hline \multirow{2}{*}{$\begin{array}{c}\text { Release rates of } \\
\text { acetic acid }(\mu \mathrm{g} / \mathrm{min})\end{array}$} & Acetic acid & $\begin{array}{c}\text { Sprayed vinegar } \\
\text { headspace }^{\mathrm{a}}\end{array}$ & $\begin{array}{c}\text { Headspace of } \\
\text { bubbled vinegar }^{\mathrm{a}}\end{array}$ \\
\hline blank & $2 \pm 1$ & $2 \pm 1$ & $1 \pm 2$ \\
0.00174 & $\mathrm{nt}^{\mathrm{b}}$ & $2 \pm 3$ & $4 \pm 5$ \\
0.0174 & $\mathrm{nt}$ & $\mathbf{4 2} \pm 14$ & $\mathbf{2 1} \pm 18$ \\
0.174 & $\mathrm{nt}$ & $\mathbf{5 1} \pm 19$ & $\mathbf{2 1} \pm 8$ \\
1.74 & $6 \pm 7$ & $\mathbf{6 9} \pm 15$ & $\mathbf{7 0} \pm 7$ \\
17.4 & $\mathbf{1 9} \pm 5$ & $\mathbf{7 4} \pm 14$ & $\mathbf{6 2} \pm 10$ \\
174 & $13 \pm 6$ & $\mathrm{nt}$ & $\mathrm{nt}$ \\
\hline
\end{tabular}

${ }^{\text {a }}$ Percentages in bold-faced font ( $N=5$ batches of 20 flies) are significantly different from the blank $(t$-test; $P<0.005)$. Sprayed air filter collections of vinegar headspace were diluted with methanol to contain known amounts of acetic acid. Bubbled vinegar from a wash bottle was diluted in water; vinegar headspace contained acetic acid at $17.4 \mu \mathrm{g} / \mathrm{min} .^{\mathrm{b}} \mathrm{nt}=$ not tested.

Antagonistic Effect of an Off-flavor on Attraction to Vinegar in the Wind Tunnel During preliminary trapping tests with the banana-water mixture brew (see below), we regularly observed a decrease in attraction when the bait was growing moldy. Geosmin, an off-flavor produced by mold fungi, had an antagonistic effect on attraction to vinegar. Vinegar headspace containing geosmin in a ratio of 1:10 (w/w) relative to acetic acid, sprayed at release rates of 0.174 and $0.0174 \mu \mathrm{g} / \mathrm{min}$ acetic acid, attracted fewer flies (18 $\pm 12 \%$ and $17 \pm 10 \%$, respectively) than vinegar without geosmin (51 and $42 \%$ attraction, respectively; Table 3$)(t$-test, $t=3.30$ and $3.28, \mathrm{df}=8, P<0.05)$.

Effect of Age, Starvation, Sex, and Mating State on Response of D. melanogaster in the Wind Tunnel The response of fed flies, disregarding age, was not significantly different from a blank test with water (Fig. 2a). Starvation during one day significantly increased the rate of upwind attraction of 1-d-old flies to the vinegar odor source. Attraction of older, 2- to 6-d-old flies peaked when they were deprived of food during $2 \mathrm{~d}$. During $24 \mathrm{hr}$ after eclosion, very few flies were attracted $(1.6 \%$, 
Upwind Attraction to Vinegar Headspace
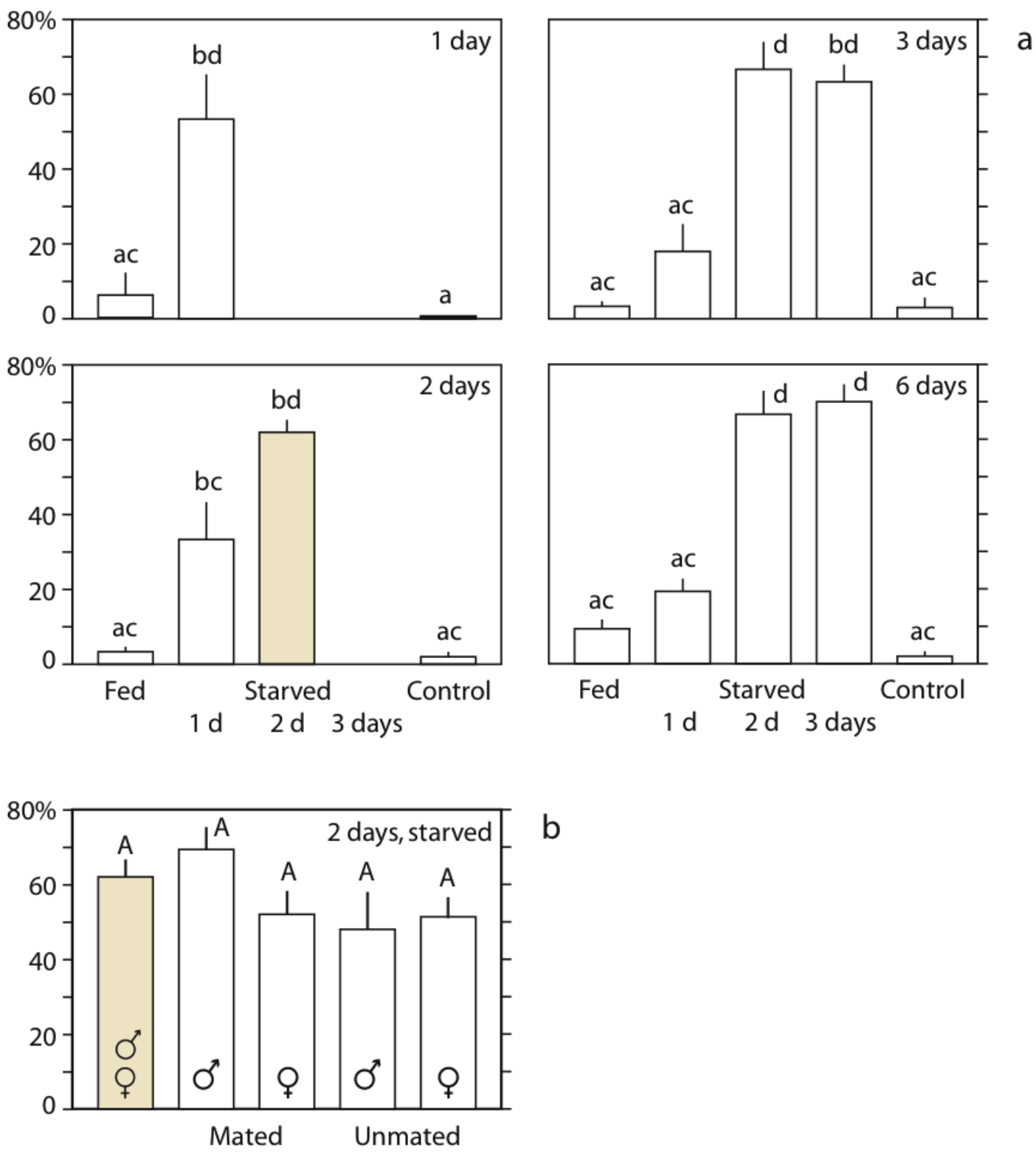

Fig. 2 Attraction of Drosophila melanogaster adults in a wind tunnel to vinegar headspace volatiles. (a) Mean percentage of 18 to 24 flies $(N=5)$ flying upwind and landing at the outlet of an air stream passing a wash bottle with vinegar. Flies, which were tested between 1 and $6 \mathrm{~d}$ after eclosion, were fed, or starved for 1 to $3 \mathrm{~d}$. For each age, water was tested as control on flies of similar starvation time as those flies showing the highest attraction to vinegar. Tests were done with a blend of males and females of unknown mating status. The shaded bar indicates conditions chosen as standard for the other wind tunnel experiments of the study. Bars with different letters are significantly different (ANOVA followed by Tukey's test; $F=20.62$; $\mathrm{df}=84, P<0.05$; error bars show standard deviation of the mean). (b) Attraction of 2d-old, 2-d-starved males and females, unseparated (shaded as shown in panel a), and sexes separated, before and after mating (ANOVA followed by Tukey's test; $F=1.54$, $\mathrm{df}=24, P=0.23$ ). 
data not shown). With flies of $1 \mathrm{~d}$ and older, age did not have a significant effect on upwind attraction (Fig. 2a). These tests were done with mixed batches of females and males, of unknown mating status. In order to verify a possible effect of sex and mating, virgin or mated flies were separated by sex either immediately after eclosion (virgin test flies) or $1 \mathrm{~d}$ before the experiment (mated test flies). Neither sex nor mating status had a significant effect on attraction (Tukey's test following ANOVA, $F=1.54, \mathrm{df}=24, P=0.23$ ) (Fig. 2b).

Trapping Study of Diel Flight Period in the Laboratory After D. melanogaster were released from their food vials, traps baited with vinegar or banana-water mixture captured $15 \%$ of the flies during the remaining $4 \mathrm{hr}$ of the photoperiod. On the following day, $39 \%$ and $62 \%$ of the remaining flies were captured, during the first and second half of the photophase, respectively. Significantly more flies were attracted to vinegar ( $76 \%$ and $72 \%$ of the trapped males and females) than to the banana-water mixture $(t$-test, $t=3.72, \mathrm{df}=10, P<0.005)$. The ratio between trapped males and females was not different ( $t$-test, $t=1.71, \mathrm{df}=4, P=0.16)$. Most flies were trapped during the photophase, only $6 \%$ of the flies were caught during the scotophase.

\section{Discussion}

Flies and other insects rely on odors to detect food and mates. Perception of odors has been thoroughly studied in D. melanogaster, particularly by extracellular recordings from olfactory neurons (De Bruyne et al. 2001; Stensmyr et al. 2003; Hallem and Carlson 2006). These neurons generate excitation patterns that are transmitted to higher brain centers where a behavioral response is created (Jefferis et al. 2007; Schlief and Wilson 2007; Shang et al. 2007; Root et al. 2008). A current challenge with $D$. melanogaster is to relate chemosensory perception and coding to the natural behavior of long-range attraction to odors.

Airborne odor is typically encountered in intermittent bursts (Baker et al. 1998) and the generation of an appropriate behavioral decision requires instantaneous assessment of its quality. Since long-range displacement costs energy and involves 
risks, it is vital to evaluate odor quality downwind from the source. Wind tunnel assays monitor the conspicuous outcome of this sensory evaluation - upwind flight orientation behavior, which is elicited within short time intervals after perception of relevant signals. We have established a sensitive and discriminative flight tunnel assay to facilitate the identification of behaviorally relevant odors, providing a link between the neurophysiology, behavioral physiology, and chemical ecology of $D$. melanogaster.

Balsamic vinegar is a robust stimulus for studying the fly's odor-mediated upwind flight attraction. Following chemical analysis, we applied the wind tunnel assay to reduce the chemical complexity of vinegar headspace (Zeppa et al. 2002; Guerrero et al. 2007; Cocchi et al. 2008) to a simple blend of volatiles that produced nearly the same attraction response as vinegar. Acetic acid, the main component of vinegar headspace, attracted flies as a single compound. Adding 2-phenyl ethanol and acetoin had a synergistic effect (Fig. 1, Table 2). This is in accordance with recent findings that the complex odor of apple cider vinegar activates several olfactory glomeruli in the antennal lobe; only two glomeruli (and consequently few odor components) are required for close-range attraction in D. melanogaster (Semmelhack and Wang 2009).

Overripe mango, which is another powerful attractant for D. melanogaster, also releases acetic acid and 2-phenyl ethanol. A blend of these two compounds with ethanol is a most attractive trap lure for D. melanogaster (Zhu et al. 2003), and addition of ethanol to the 3- and 4-component synthetic blends that we tested in our study might complement the attraction found for the complete vinegar bouquet. Thus, the role of ethanol in the long-range attractant for D. melanogaster merits additional study.

Attraction to acetic acid is not unique to D. melanogaster. Also noctuid moths that are attracted to fermenting sweet baits respond to acetic acid (Landolt 2000). 2Phenyl ethanol is, like acetic acid, a yeast product; it is also found in insectpollinated plants and known to attract many insect species from different taxa (Andersson et al. 2002; El-Sayed 2009).

Sensitivity to acetic acid is expressed in D. melanogaster adults and larvae (Hoffmann and Parsons 1984; Cobb 1999; Ruebenbauer et al. 2008; Joseph et al. 
2009), supporting the idea that this compound is of ecological relevance. Acetic acid probably serves as a cue for the presence of fermenting fruit and other substrates used as food or oviposition sites.

Interestingly, Joseph et al. (2009) recently demonstrated gustatory-mediated attraction vs. olfactory-mediated positional repulsion in response to egg-laying substrates containing acetic acid, under close-range conditions. Under long-range conditions acetic acid plays an essential role in upwind flight attraction (Table 2), which we assume is relayed through olfactory neurons. Despite its strong behavioral effect, it is yet unclear how D. melanogaster perceives and processes acetic acid. In comparison, olfactory neurons expressing receptors for 2-phenyl ethanol, acetoin and ethyl acetate and their associated glomeruli in the antennal lobe are already known (Fishilevich and Vosshall 2005; Hallem and Carlson 2006; De Bruyne and Baker 2008; Asahina et al. 2009).

Semmelhack and Wang (2009) showed that activity in the glomeruli DM1 and VA2 is associated with close-range attraction of D. melanogaster to vinegar. The vinegar compounds ethyl acetate and acetoin are the strongest known stimuli for these two glomeruli (De Bruyne and Baker 2008). Ethyl acetate and acetoin may be sufficient for close range attraction and oviposition (Ruebenbauer et al. 2008; Semmelhack and Wang 2009), but a blend of these compounds did not induce upwind flight attraction in the absence of other vinegar volatiles (Table 2). It will be rewarding to study the representation of defined blends of synthetic compounds in comparison with complex authentic odors such as vinegar.

The threshold concentration for initiation of upwind flight reflected odor quality. D. melanogaster responded to the odor of vinegar even at a 1000 -fold dilution, or a release rate of $17.4 \mathrm{ng} / \mathrm{min}$ of the main compound acetic acid. In comparison, attraction to a 10-fold dilution of acetic acid alone was not significant (Table 3). Admixture of further vinegar volatiles to acetic acid had a synergistic effect on attraction (Table 2), while admixture of geosmin, an off-flavor produced by microorganisms such as mold fungi (La Guerche et al. 2006) produced an opposite, antagonistic effect on attraction to vinegar.

The stimulus application method is a keystone element of wind tunnel bioassays. The piezoelectric sprayer (El-Sayed et al. 1999) enables the release of chemicals at a 
known constant rate and purity. It enables the parallel chemical and behavioral analysis of headspace collections of natural odour sources, as a starting point for tests with synthetic chemicals. The comparison of fly attraction to authentic and revaporized headspace corroborates the validity of the spray application procedure (Fig. 1).

The piezoelectric sprayer disperses solutions of synthetic and authentic chemicals in solvent. Methanol, which did not elicit a significant response from $D$. melanogaster (Table 1), is a good choice for a solvent in this spray system. It is easily vaporized and dissolves polar- and to some extent, non-polar compounds. Our results on the response to methanol agree with a study by Hoffmann and Parsons (1984), showing no attraction of D. melanogaster and three other species of Drosophila to methanol. Comparison between authentic vinegar- and sprayed headspace samples did not show an impact by methanol (Fig. 1c). Interestingly, tests on higher dilutions of vinegar headspace $(1: 100$ and 1:1000) indicated a stronger attraction to the methanol-diluted sprayed headspace than to the vinegar that was diluted with distilled water (Table 3). This suggests a possible synergistic activity of methanol to stimuli tested at threshold concentrations, and the effect of methanol in the context of other components of the attractant bears further investigation. It is, however, unclear if this effect was due to the solvent or due to the different odor application methods.

Water is a straightforward solvent for vinegar however dilutions in water cannot be analyzed by gas chromatography. It is remarkable that a few flies responded to distilled water, although attraction was not significantly different from blank (Table 1). Water has earlier been reported to be critically important for attraction to traps baited with synthetic fruit odors (Zhu et al. 2003). Drosophila melanogaster sense water with gustatory receptor neurons on the proboscis, projecting into a specific region of the suboesophagal ganglion, and with hygrosensory neurons located on the antennae, projecting into the antennal mechanosensory centre (Fischler et al. 2007; Liu et al. 2007; Inoshita and Tanimura 2008). It is remarkable that stimuli from outside the antennal lobe, the olfactory center, generate or contribute to an upwind flight response in D. melanogaster. 
The response of D. melanogaster to vinegar was modulated by hunger, while sex and age did not have an effect (Fig. 2). The olfactory system is under circadian clock control (Tanoue et al. 2008), sexual activity peaks during the night (Fujii et al. 2007), but flies responded to food during the day. Starvation had a decisive effect on attraction to vinegar, in both sexes, irrespective of mating state. Upwind flight of hungry flies to the odor of vinegar demonstrates that odor cues are processed with respect to the physiological state of the fly to generate an appropriate behavioral response. In case of a hungry fly, this response is expressed as a voluntary take off and the initiation of flight towards the odor source.

We conclude that our wind tunnel assay and the piezoelectric delivery system enables the measurement of a conspicuous and unambiguous behavioral response. The measurement of this response provides the necessary resolution for the investigation of physiologically and ecologically relevant odors and will become an essential tool for the functional analysis of the D. melanogaster olfactory system.

Acknowledgments This study was supported by the Linnaeus initiative "Insect Chemical Ecology, Ethology and Evolution” IC-E3 (Formas, SLU). We thank Teun Dekker and Mattias Larsson for helpful comments and discussions. Jörgen Lantz constructed the wind tunnel.

\section{References}

ALlEN, M. J., GODENSCHWEGE, T. A., TANOUYE, M. A., and PHELAN, P. 2006. Making an escape: development and function of the Drosophila giant fibre system. Semin. Cell Dev. Biol. $17: 31-41$.

ANDERSSON, S., NILSSON, L. A., GROTH, I., and BERGSTRÖM, G. 2002. Floral scents in butterfly-pollinated plants: possible convergence in chemical composition. Bot. J. Linn. Soc. 140:129-153.

ASAHINA, K., LOUIS, M., PICCINOTTI, S., and VOSSHALL, L. B. 2009. A circuit supporting concentration-invariant odor perception in Drosophila. J. Biol. 8:9.

BAKER, T. C., FADAMIRO, H. Y., and COSSÉ, A.A. 1998. Moth uses fine tuning for odor resolution. Nature 393:530.

BARTELT, R. J., SCHANER, A. M., and JACKSON, L. L. 1985. cis-Vaccenyl acetate as an aggregation pheromone in Drosophila melanogaster. J. Chem. Ecol. 11:1747-1756.

BENTON, R., VANNICE, K. S., and VOSSHALL, L. B. 2007. An essential role for a CD36-related receptor in pheromone detection in Drosophila. Nature 450:289-293. 
BUDICK, S. A. and DICKINSON, M. H. 2006. Free-flight responses of Drosophila melanogaster to attractive odors. J. Exp. Biol. 209:3001-3017.

CARD, G. and DICKINSON, M. H. 2008. Visually mediated motor planning in the escape response of Drosophila. Current Biology 18:1300-1307.

CHOW, D. M. and FRYE, M. A. 2008. Context-dependent olfactory enhancement of optomotor flight control in Drosophila. J. Exp. Biol. 211:2478-2485.

COBB, M. 1999. What and how do maggots smell? Biol. Rev. 74:425-459.

COCCHI, M., DURANTE, C., GRANDI, M., MANZINI, D., and MARCHETTI, A. 2008. Three-way principal component analysis of the volatile fraction by HS-SPME/GC of aceto balsamico tradizionale of modena. Talanta 74:547-554.

COUTO, A., ALENIUS, M., and DICKSON, B. J. 2005. Molecular, anatomical, and functional organization of the Drosophila olfactory system. Current Biology 15:1535-1547.

DE BRUYNE, M., FOSTER, K., and CARLSON, J. R. 2001. Odor coding in the Drosophila antenna. Neuron 30:537-552.

DE BRUYNE, M. and BAKER, T. C. 2008. Odor detection in insects: volatile codes. J. Chem. Ecol. 34:882-897.

DICKSON, B. J. 2008. Wired for sex: the neurobiology of Drosophila mating decisions. Science 322:904-909.

EL-SAYED, A. M. 2009. The Pherobase: Database of Insect Pheromones and Semiochemicals. http://www.pherobase.com/

EL-SAYED, A., GÖDDE, J., and ARN, H. 1999. Sprayer for quantitative application of odor stimuli. Environ. Entomol. 28:947-953.

FISCHLER, W., KONG, P., MARELLA, S., and SCOTT, K. 2007. The detection of carbonation by the Drosophila gustatory system. Nature 448:1054-1058.

FISHILEVICH, E., and VOSSHALL, L. B. 2005. Genetic and functional subdivision of the Drosophila antennal lobe. Current Biology 15:1548-1553.

FUJII, S., PARTHASARATHY, K., HARDIN, P., and AMREIN, H. 2007. Nocturnal male sex drive in Drosophila. Current Biology 17:244-251.

GUERRERO, E. D., MARIN, R. N., MEJIAS, R. C., and BARROSO, C. G. 2007. Stir bar sorptive extraction of volatile compounds in vinegar: validation study and comparison with solid phase microextraction. J. Chromatogr. A 1167:18-26.

HALLEM, E. A. and CARLSON, J. R. 2006. Coding of odors by a receptor repertoire. Cell 125:143160.

HOFFMANN, A. A. and PARSONS P. A. 1984. Olfactory response and resource ulilization in Drosophila: interspecific comparisons. Biolog. J. Lin. Soc. 22: 43-53.

INOSHITA, T. and TANIMURA, T. 2008. Cellular identification of water gustatory receptor neurons and their central projection pattern in Drosophila. Proc. Ntl. Acad. Sci. USA 103:1094-1099.

JEFFERIS, G. S. X. E., POTTER, C. J., CHAN, A. M., MARIN, E. C., ROHLFING, T., MAURER, C. R., and LUO, L. 2007. Comprehensive maps of Drosophila higher olfactory centers: Spatially segregated fruit and pheromone representation. Cell 128:1187-1203.

JOSEPH, R. M., Devineni, A., King, I. F. G., and Heberlein U. 2009. Oviposition preference for and positional avoidance of acetic acid provide a model for competing behavioral drives in Drosophila. PNAS 106:11352-11357. 
LA GUERCHE, S., DAUPHI, B., PONS, M., DOMINIQUE, B., and DARRIET, P. 2006. Characterization of some mushroom and earthy off-odors microbially induced by the development of rot on grapes. J. Agric. Food. Chem. 54:9193-9200.

LANDOLT, P. J. 2000. New chemical attractants for trapping Lacanobia subjuncta, Mamestra configurata, and Xestia c-nigrum (Lepidoptera: Noctuidae). J. Econ. Entomol. 93:101-106.

LIU, L., LI, Y., WANG, R., YIN, C., DONG, Q., HING, H., KIM, C., and WELSH, M. J. 2007. Drosophila hygrosensation requires the TRP channels water witch and nanchung. Nature 450:294-299.

ROOT, C. M., MASUYAMA, K., GREEN, D. S., ENELL, L. E., NÄSSEL, D. R., LEE, C.-H., and WANG, J. W. 2008. A presynaptic gain control mechanism fine-tunes olfactory behavior. Neuron 59:311-321.

RUEBENBAUER, A., SCHLYTER, F., HANSSON, B. S., LÖFSTEDT, C., and LARSSON, M. C. 2008. Genetic variability and robustness of host odor preference in Drosophila melanogaster. Current Biology 18:1438-1443.

SCHLIEF, M. L. and WILSON, R. I. 2007. Olfactory processing and behavior downstream from highly selective receptor neurons. Nature Neurosc. 10:623-630.

SEMMELHACK, J. L. and WANG, J. W. 2009. Select Drosophila glomeruli mediate innate olfactory attraction and aversion. Nature 459:218-224.

SHANG, Y., CLARIDGE-CHANG, A., SJULSON, L., PYPAERT, M., and MIESENBÖCK, G. 2007. Excitatory local circuits and their implications for olfactory processing in the fly antennal lobe. Cell 128:601-612.

STENSMYR, M. C., GIORDANO, E., BALLOI, A., ANGIOY, A. M., and HANSSON, B. S. 2003. Novel natural ligands for Drosophila olfactory receptor neurones. J. Exp. Biol. 206:715-724.

TANOUE, S., KRISHNAN, P., CHATTERJEE, A., and HARDIN, P. E. 2008. G protein-coupled receptor kinase 2 is required for rhythmic olfactory responses in Drosophila. Current Biology 18:787-794.

TASIN, M., BÄCKMAN, A.-C., BENGTSSON, M., VARELA, N., IORIATTI, C., and WITZGALL, P. 2006. Wind tunnel attraction of grapevine moth females, Lobesia botrana, to natural and artificial grape odour. Chemoecology 16:87-92.

TRIMARCHI, J. R. and SCHNEIDERMAN, A. M. 1995. Different neural pathways coordinate Drosophila flight initiations evoked by visual and olfactory stimuli. J. Exp. Biol. 198:10991104.

VOSSHALL, L. B. and STOCKER, R. E. 2007. Molecular architecture of smell and taste in Drosophila. Ann. Rev. Neurosci. 30:505-533.

ZEPPA, G., GIORDANO, M., GERBI, V., and MEGLIOLI, G. 2002. Characterisation of volatile compounds in three acetification batteries used for the production of "Aceto Balsamico Tradizionale di Reggio Emilia". Ital. J. Food Sci. 14:247-266.

ZHU, J., PARK, K.-C., and BAKER, T. C. 2003. Identification of odors from overripe mango that attract vinegar flies, Drosophila melanogaster. J. Chem. Ecol. 29:899-909. 